\title{
Study on Insertion Loss of Fiber Fabry-Perot Filters
}

\author{
Sheng-Nan Su, Hai-Bing Qi, Yong-Lin Yu* \\ Wuhan National Laboratory for Optoelectronics, Huazhong University of Science and Technology, Wuhan, China. \\ Email: *yonglinyu@mail.hust.edu.cn
}

Received October 2013

\begin{abstract}
Fiber Fabry-Perot (FFP) filters have been widely used in optical fiber communications, and insertion loss (IL) is one of its important characteristics. Based on theoretically analysis, factors related to IL were discussed. In order to investigate the IL of different structures, simulations are carried out with finite-difference time-domain (FDTD) algorithm. Comparisons are made between the optimized structure and full-size-mirror FFP filter, and fiber-inserted FFP filter as well. Simulation results demonstrated that the finite-size-mirror structure can confine the mode size of the open resonator, hence reduce the IL of FFP filter.
\end{abstract}

Keywords: FFP Filter; Insertion Loss; Finesse

\section{Introduction}

Tunable fiber Fabry-Perot filters (FFP-TFs) are widely used in optical fiber communications and optical sensing systems, since they have advantages of low loss, high tuning speed, wide tuning range, high finesse, and flexible structures and different practical applications. For optical fiber communication applications, FFP-TFs can be used to select wavelength for each channel in wavelength division multiplexing (WDM) system [1]. In fiber Brag grating (FBG) sensing systems, FFP-TFs can be adopted in dynamically demodulation [2]. With the help of a FFP-TF, wavelength tuning of an optical fiber ring laser could be realized [3]. FFP-TFs also find applications in clock signal extraction in all optical 3R regeneration system and optical network unit [4,5]. Recently, applications of FFP-TFs in microwave photonic system have drawn some attentions $[6,7]$.

Insertion loss is one of the important characteristics of FFP-TFs. Insertion loss is classified into two kinds of loss, namely extrinsic loss and intrinsic loss. The extrinsic loss of FFP-TFs can be reduced by improvement of device manufacturing process and assembling preicision. Reducing the intrinsic loss of FFP-TFs, however, needs to optimize their structures. For this purpose, a lot of work has been reported. In1989, D. Marcuse from Bell labs and MOI, USA, proposed a FFP-TF structure with a inserted waveguide [8,9]. In 2005, Y. Bao et al. from MOI, USA, patented a FFP-TF structure with a concave cavity [10]; In 2011, Fujikawa et al. from Tokyo Univer-

"Corresponding author. sity, Japan, presented a structure with an expanded core fiber [11]. In 2008, C. Tang et al. from Beijing Institute of Technology put forward a structure with convex lens after the mirrors [12]. In 2009, we proposed a new structure based on finite design [13].

In this paper, we begin with fundamental analysis of insertion loss of FFP-TFs, and discuss effects of key parameters of the structure on the insertion loss. Then we focused on our new finite-size-mirror structure. Characteristics and mode distribution of the finite-size-mirror FFP-TF are studied and simulated. Finally we compare this new finite-size-mirror structure with fiber inserted structure and full-size-mirror structure.

\section{Basic Principle}

The FFP filter is based on multiple beam of F-P cavity. As is shown in Figure 1(a), FFP filter which consists of two aligned fiber coating with high reflecting film. Assuming that the light enters the F-P cavity with an angle $(\theta)$ against the normal, the light beams exit the second fiber parallelly after multiple reflecting, as is shown in Figure 1(b). The parallel beams are amplitude decreased and superposed coherent.

The intensity $\left(\mathrm{I}^{(t)}\right)$ of the output light is,

$$
I^{(t)}=\frac{T_{m}^{2}}{\left(1-R_{m}\right)^{2}+4 R_{m} \sin ^{2}(\delta / 2)} I^{(i)} .
$$

$I^{(i)}$ is the intensity of input light, $R_{m}$ is the reflectivity of the coated film, $T_{m}$ is the transmission of the mirror. $\delta=4 \pi n d \cos \theta / \lambda$ is the phase difference of the 


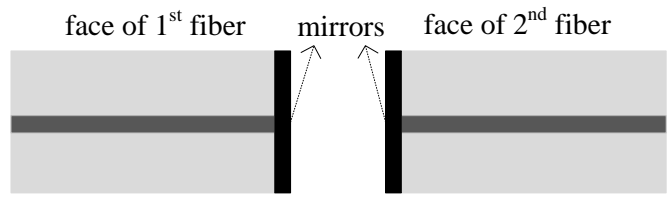

(a) The structure of a FFP filter

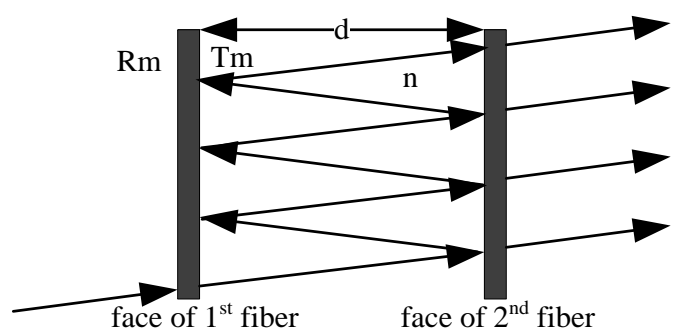

(b) The principle of FFP filter

Figure 1. The structure of FFP filter (a) and the principle of FFP filter (b).

adjacent parallel beams, $\mathrm{n}$ is the refractive index of the medium within the F-P cavity, $\mathrm{d}$ is the length of the F-P cavity and $\lambda$ is the central wavelength of the input light. The wavelength which meets

$\delta=4 \pi n d \cos \theta / \lambda=2 \mathrm{~m} \pi$ will exit FFP filter. Obviously, tuning the transmission wavelength can be achieved by changing the parameters related to the phase difference. The transmittance of the FFP filter, is defined as the ratio of $\mathrm{I}^{(\mathrm{t})}$ to $\mathrm{I}^{(\mathrm{i})}$, that is,

$$
\mathrm{T}_{\mathrm{cav}}=\frac{\mathrm{T}_{\mathrm{m}}^{2}}{\left(1-\mathrm{R}_{\mathrm{m}}\right)^{2}+4 \mathrm{R}_{\mathrm{m}} \sin ^{2}(\delta / 2)} .
$$

When phase difference $\delta=2 n \pi$, FFP filter attains maximum transmittance, namely peak transmittance ( $\mathrm{T}$ ). Insertion loss (IL) of a FFP filter is then expressed as,

$$
\mathrm{IL}=-10 \lg \left(\frac{\mathrm{I}^{(\mathrm{i})}-\mathrm{I}_{\max }^{(\mathrm{t})}}{\mathrm{I}^{(\mathrm{i})}}\right)=-10 \lg (\mathrm{T}) .
$$

The finesse (F) of FFP filter is, $F=\pi \sqrt{R_{m}} /\left(1-R_{m}\right)$, despite of loss (L). When considering loss, the peak transmittance is [14],

$$
\mathrm{T}=1 /\left(1+\mathrm{L} / \mathrm{T}_{\mathrm{m}}\right)^{2} \text {. }
$$

If the loss is ultra small, the finesse is then expressed as follow,

$$
\mathrm{F}=\pi /\left(\mathrm{L}+\mathrm{T}_{\mathrm{m}}\right)
$$

From the above analysis, it is obviously that low IL is necessary if high peak transmittance and high finesse is required.

Factors leading to insertion loss of FFP filter can be classified into two kinds, extrinsic loss and intrinsic loss, according to the method to classify the splice loss of single mode fiber [16]. Extrinsic loss is generated during the manufacturing process, including loss dues to fiber tilt and offset. Intrinsic loss is caused by the structure of FFP filter. It includes diffraction loss and mode mismatch loss between guided wave mode and open mode of F-P cavity. Improvement of the device manufacturing and assembly precision will reduce the extrinsic loss. However, reducing the intrinsic loss needs to optimize the structure of FFP filter.

\section{Fundamental Analysis on Insertion Loss}

Transmittance coefficient $\left(T_{f}\right)$ of single mode fiber with longitudinal separation can be calculated with [17],

$$
\mathrm{T}_{f}=\frac{4\left[4 \mathrm{Z}^{2}+\frac{\omega_{1}{ }^{2}}{\omega_{2}{ }^{2}}\right]}{\left[4 \mathrm{Z}^{2}+\frac{\omega_{1}{ }^{2}+\omega_{2}{ }^{2}}{\omega_{2}{ }^{2}}\right]+4 \mathrm{Z}^{2} \frac{\omega_{1}{ }^{2}}{\omega_{2}{ }^{2}}} .
$$

$\omega$ is the mode field radius two optical fibers respectively, $\mathrm{Z}=\mathrm{d} / E \omega_{1} \omega_{2}$ is the normalized fiber separation distance, $k$ is the free space wave number. Consider that the input field bounces back and forth in the FFP filter's resonator, it traverses an effective length that is defined by the mirror transmission [8],

$$
\mathrm{L}_{E}=2 \mathrm{~d} / \mathrm{T}_{\mathrm{m}} \text {. }
$$

In a simple-minded view, we regard the transmittance of FFP filter as a splice of identical fibers with an end separation equal to the effective length. Suppose,

$$
\mathrm{k} \omega_{0}^{2} \mathrm{~T}_{\mathrm{m}} / 2 \mathrm{~d}=\mathrm{x}=\mathrm{k} \omega_{0}^{2} / \mathrm{L}_{\text {eff }}=1 / \mathrm{Z},
$$

then

$$
\mathrm{T}=\frac{\mathrm{x}^{2}}{1+\mathrm{x}^{2}} .
$$

This expression is plotted in Figure 2.

It is apparently that the peak transmittance increases with the increasing of $x$. Equation (8) shows that expanding the fiber mode field radius, increasing reflectiv-

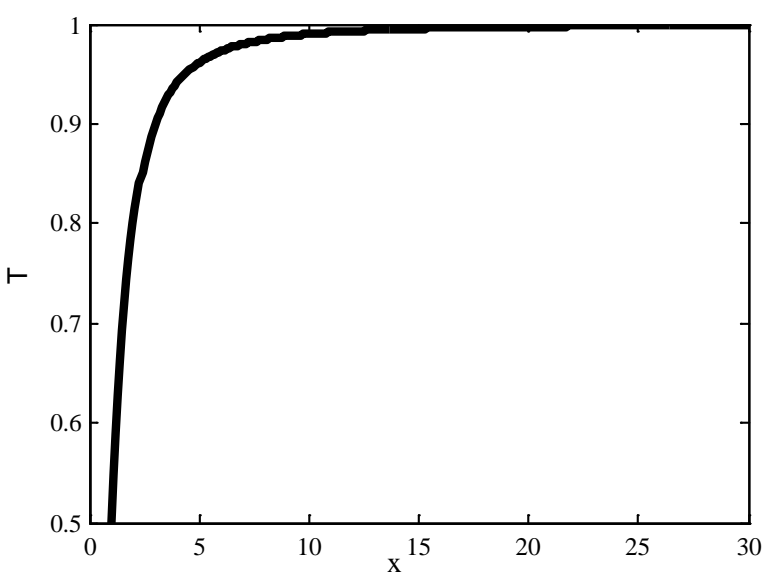

Figure 2. The peak transmittance varies with $x$. 
ity and shorten cavity length could enlarge x. Large mode field radius will reduce the mode mismatching loss, as a result, the peak transmittance increases. Improving the transmittance of mirror or shorten the cavity length will also increase peak transmittance of FFP filter, cause the shorter the length is, the less diffraction loss there will be. All these relationships are plotted in Figure 3.

\section{Finite-Size-Mirror Structure}

For the purpose of reduction of IL, we proposed a new structure, named a Finite-size-mirror structure [13]. As is shown in Figure 4, we use a finite mirror substituting the traditional mirror with a full-fiber-end covering mirror substituting traditional mirror. In this new structure, mirrors only cover part of fiber ends. Mode size of open resonator is proportion to its size [18], so smaller mode size can be attained with short radius of mirror. Nonetheless, short radius mirrors increase diffraction loss. Finite-size-mirror structure can be worked on condition that an optimal radius of mirror is found out.

Relation of transmittance with radii of mirrors of the FFP-TF is investigated with the help of finite difference time domain (FDTD) algorithm. The mirror reflectivity is set as 0.697 , and cavity length is set as $9 \mu \mathrm{m}$. As a result, relationship between peak transmittance and radius of mirror is shown in Figure 5.

Figure 5 clearly shows that the peak transmittance of FFP-TF firstly increases with the increasing radius of the mirrors. However, it decreases when the radius of mirrors increase to a certain value (about $6 \mu \mathrm{m}$ ), and then it tends to be a constant when the radius of mirrors further increases $(>12 \mu \mathrm{m})$. Figure 5 indicates that there is a lowest value of the IL of FFP-TF, corresponding to the maximum value of the peak transmittance.

\section{Simulations and Comparisons}

We analyzed key parameters and output mode field and compared finite-size-mirror structure with other two structures to study more about finite-size-mirror FFP-TF. Here, three structures are investigated as shown in Figure 6. Figure 6(a) shows typical structure with full size mirrors, Figure 6(b) represents a fiber inserted structure, and Figure 6(c) is our structure with finite size mirrors. Simulations for the above three structures are carried out in two
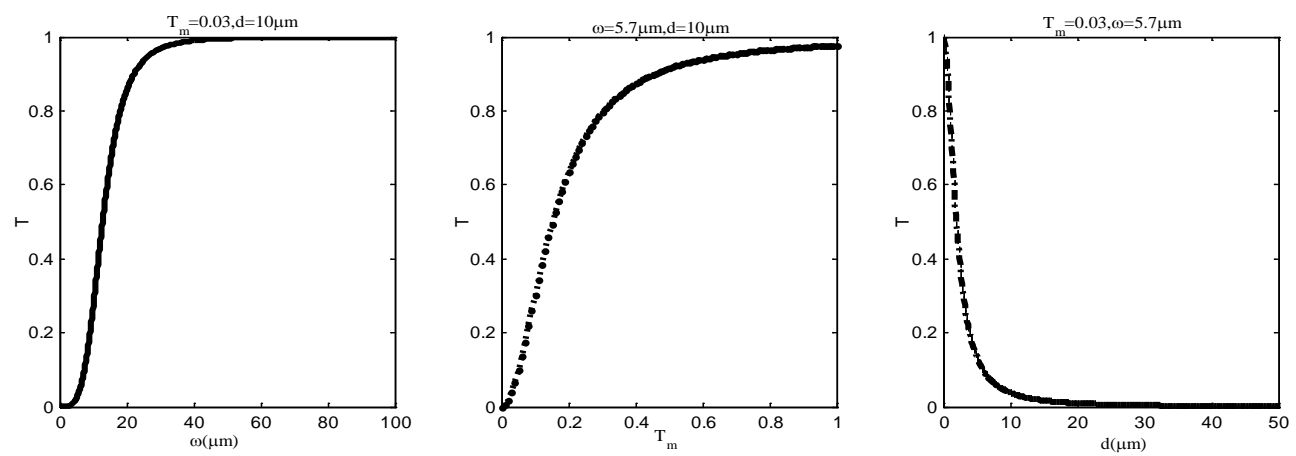

Figure 3. The peak transmittance of FFP filter varies with $\omega_{0}, T_{m}$ and $d$.

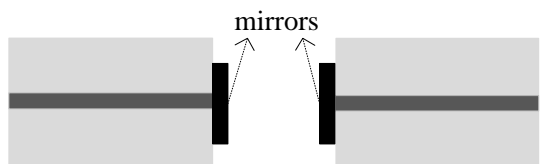

Figure 4. The structure of Finite-size-mirror FFP filter.

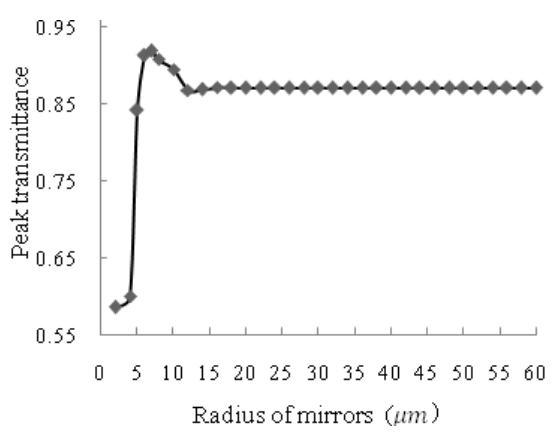

Figure 5. Peak transmittance varies with radii of mirrors.

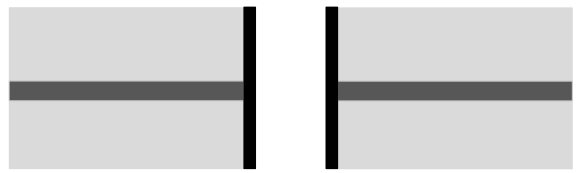

(a) Full-size-mirror FFP filter

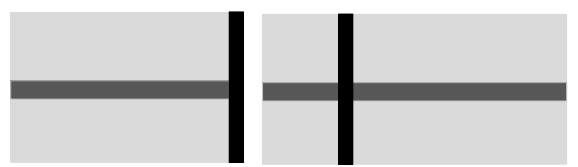

(b) Fiber inserted FFP filter

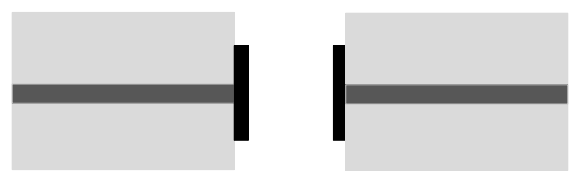

(c) Finite-size-mirror FFP filter

Figure 6. Structures of the three kinds of FFP filters. 
dimension and three dimension. Transmission spectra can be extracted from results of two dimension simulation. Peak transmittance, free spectral range and finesse can be found out from transmission spectrum. Results of three dimension simulation can be used to analyze distribution of output mode.

In the simulation, we assume that the radius of mirrors is the same as that of the fiber cladding for Figure 6(a) and Figure 6 (b), and the radius of mirror is $6 \mu \mathrm{m}$. For all cases, the reflectivity is 0.697 , the optical cavity's optical length is $9 \mu \mathrm{m}$ and central wavelength is $1.495 \mu \mathrm{m}$. Simulated transmission spectra of three FFP filter structures are shown in Figure 7. The key characteristics, such as insertion loss and finesse, are listed in Table $\mathbf{1 .}$

In Table 1, structures (a) (b) (c) correspond with three structures cases shown in Figure 6 respectively. M.R. means the radius of mirror, $\mathrm{T}$ represents peak transmittance of a FFP filter, Tmin is the valley value of spectrum. FSR and FWHM are free spectral range, full width at half maximum of spectrum separately. F and IL are finesse and insertion loss of a filter, respectively. Clearly, for Figures 6(b) and (c), there are some reduction of the insertion loss due to improvements on structure.

More intuitive results obtained by three-dimensional simulation are shown in Figure 8, which present distribution of output mode field. It can be seen that, for full-

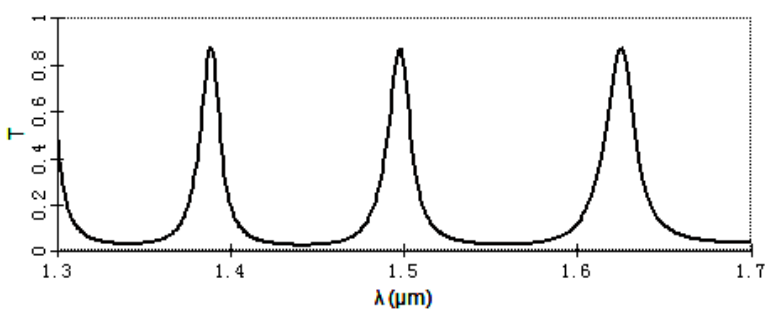

(a) Full-size-mirror FFP filter

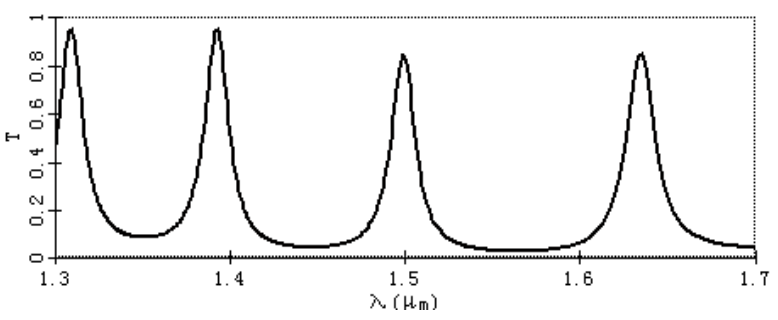

(b) Fiber inserted FFP filter

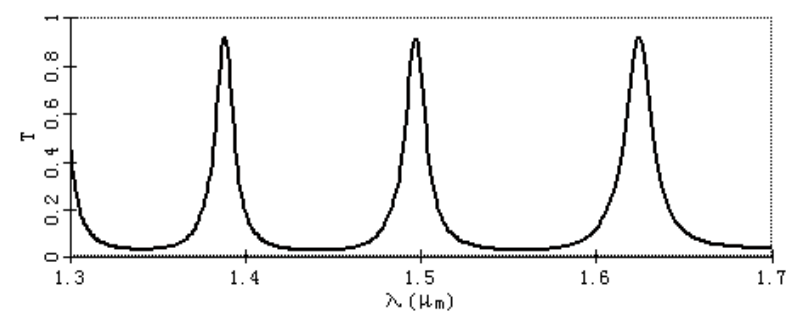

(c) Finite-size-mirror FFP filter

Figure 7. Simutlated transmission spectra of FFP filters.
Table 1. parameters of three different FFP filters.

\begin{tabular}{cccc}
\hline structures & (a) & (b) & (c) \\
\hline M.R. $(\mu \mathrm{m})$ & 60 & 60 & 6 \\
$\mathrm{~T}$ & 0.875 & 0.903 & 0.921 \\
$\mathrm{~T}_{\min }$ & 0.0311 & 0.0553 & 0.0322 \\
FSR $(\mathrm{nm})$ & 118 & 109 & 118 \\
FWHM(nm) & 20.4 & 18.0 & 14.7 \\
$\mathrm{~F}$ & 6 & 6 & 8 \\
$\mathrm{IL} / \mathrm{dB}$ & 0.580 & 0.443 & 0.357 \\
\hline
\end{tabular}

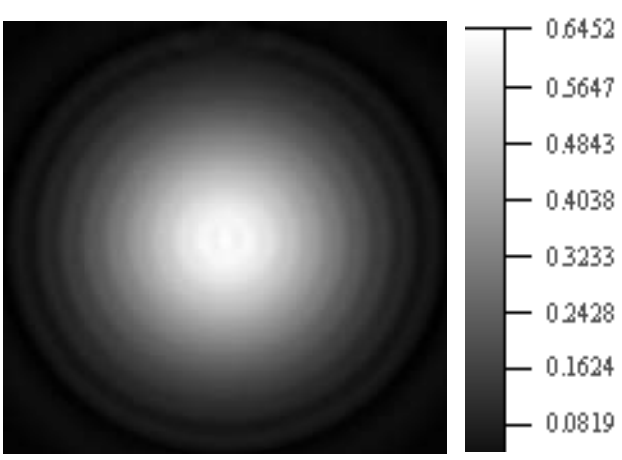

(a)Full-size-mirror FFP filter

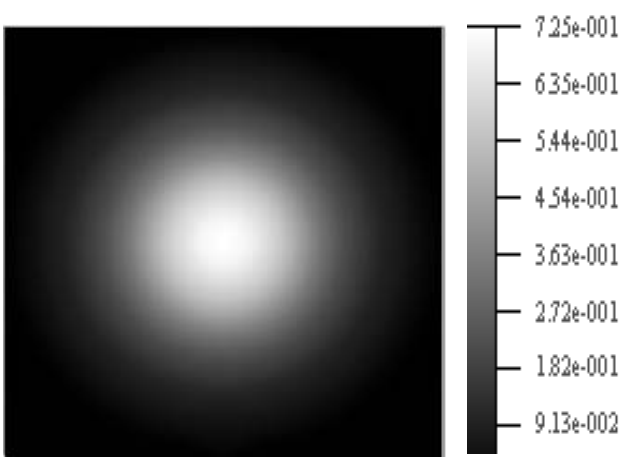

(b) Fiber inserted FFP filter

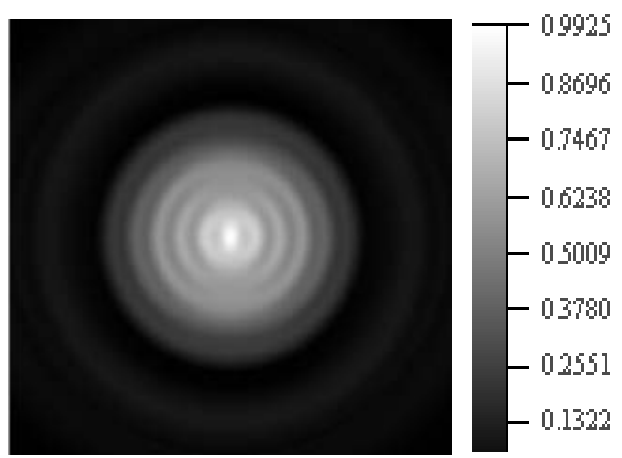

(c) Finite-size-mirror FFP filter

Figure 8. Mode field distribution of output field.

size-mirror structure and fiber inserted structure, the output field of filters distributes in the area of fiber cladding and core, while for the finite-size-mirror filter the output field concentrates in the area of fiber core, and its centre intensity is much stronger. This is due to the reduction of 
mode mismatch with limitation to the mode size of resonant cavity of finite sized mirror. The simulation results demonstrate that finite-size-mirror filter do help with reduction of insertion loss of FFP filter.

\section{Summary and Conclusions}

We have studied the insertion loss of FFP filters. Based on discussion of effects on insertion loss, finite-size-mirror FFP filter was simulated and compared.

Insertion loss is one of the important characteristics of FFP filter. Simulation results demonstrate that finite-sizemirror structure helps with reduction in insertion loss of FFP filter. This is because finite-size-mirror can limit the mode size of F-P cavity.

\section{Acknowledgements}

This work was supported in part by the Specialized Research Fund for the Doctoral Program of Higher Education (SRFDP) under Grant No. 20100142110045, and in part by the National Natural Science Foundation of China under Grant No. 11174097.

\section{REFERENCES}

[1] C M. Miller, "Passive Tunable Fiber Fabry-Perot Filters for Transparent Optical Networks,” Scientific American, 1991, p. 1.

[2] C. Zhang, C. Miao, H. Li, et al., "Design of Distributed FBG Vibration Measuring System Based on Fabry-Perot Tunable Filter,” Proceedings of SPIE, Vol. 2011, 8201, 820110.

[3] Y. Wei, K. Hu, B. Sun and T. Wang, “All-Fiber Widely Wavelength-Tunable Thulium-Doped Fiber Ring Laser Incorporating a Fabry-Perot Filter,” Laser Physics, Vol. 22, No. 4, 2012, pp. 770-773. http://dx.doi.org/10.1134/S1054660X12040263

[4] D. Petrantonakis, G. T. Kanellos, P. Zakynthinos, D. Apostolopoulos, N. Pleros and H. Avramopoulos, "40Gb/s 3R Burst Mode Regenerator Using Four Integrated MZI Switches,” IEEE Photonics Technology Letters, Vol. 19, No. 5, 2007, pp. 288-290. http://dx.doi.org/10.1109/LPT.2006.890051

[5] E. Kehayas, "Designing Wavelengthlength Divsion-Multiplexed Optical Access Networks Using Reflective Photonic Components,” ICTON, 2010.
[6] X. J. Xie, et al., "Broadband Photonic Radio-Frequency Channelization Based on a 39-GHz Optical Frequency Comb,” IEEE Photonics Technology Letters, Vol. 24, No. 8, 2012, pp. 661-663.

[7] Y. Ji, S. Zheng, Z. Li, X. Jin, X. Zhang and H. Chi, "Tunable Fiber Fabry-Perot for Pm-Im Conversion and Efficiency Improvement Inradio-Over-Fiber Links,” Microwave and Optical Technology Letters, Vol. 52, No. 9, 2010, pp. 2090-2095.

http://dx.doi.org/10.1002/mop.25389

[8] D. Marcuse and J. Stone, "Fiber-Coupled Short FabryPerot Resonators," Journal of Lightwave Technology, Vol. 7, No. 5, 1989, pp. 869-876. http://dx.doi.org/10.1109/50.19128

[9] Y. Bao, K. Hsu, T. Q. Li, C. M. Miller and J. W. Miller, "Cascaded Fixed and Tunable Multiple-Fiber Fabry-Perot Filters and Fixed and Tunable Ferrule Alignment Fixtures for Fiber Fabry-Perot filters,” US Patent 6241397, 2001.

[10] Y. Bao, S. K. Ferguson and D. Q. Snyder, "Waferless Fiber Fabry-Perot Filters,” US Patent 6904206, 2005.

[11] C. Fujikawa. T. Shintaku, et al., "Fiber Fabry-Perot Optical Cavities Using Expanded-Core Fibers with Concave Form,” 17th Microopics Conference (MOC), 30 October 2 November 2011, pp. 1-2.

[12] Y. Jiang and C. Tang, "High-Finesse Micro Lens Optical Fiber Fabry-Perot Filters,” Microwave and Optical Technology Letters, Vol. 50, No. 9, 2008, pp. 2386-2389. http://dx.doi.org/10.1002/mop.23679

[13] H. B. Qi and Y. L. Yu, "The Method to Reduce the Insertion Loss of Fiber Fabry-Perot Filters," China Patent 2009100638914, 2011. (in Chinese)

[14] M. Born and E. Wolf, "Principles of Optics," 5th Edition, Pergamon, Oxford, 1985, p. 329.

[15] J. Stone and D. Marcuse, "Ultrahigh Finesse Fiber FabryPerot Interferometers,” Journal of Lightwave Technology, Vol. 4, No. 4, 1986, pp. 382-385. http://dx.doi.org/10.1109/JLT.1986.1074739

[16] W. Q. Li, "Several Factors of Fiber Connecting Loss," Telecommunications for Electric Power System, Vol. 2, 1999, pp. 37-38. (in Chinese)

[17] D. Marcuse,” Loss Analysis of Single-Mode Fiber Splices,” Bell System Technical Journal, Vol. 56, No. 5, 1977, pp. 703-718. http://dx.doi.org/10.1002/j.1538-7305.1977.tb00534.x

[18] H. Kogelnik and T. Li, "Laser Beams and Resonators," Proceedings of IEEE, Vol. 54, No. 10, 1966, pp. 13121329. http://dx.doi.org/10.1109/PROC.1966.5119 